Chirurg 2013 $\cdot 84: 525$

DOI 10.1007/s00104-013-2520-1

Online publiziert: 18. Mai 2013

(c) Springer-Verlag Berlin Heidelberg 2013

O. Strobel · M.W. Büchler

Klinik für Allgemein-, Viszeral- und Transplantationschirurgie, Universität Heidelberg

\title{
Kein Vorteil für die routinemäßige Drainage nach Pankreasresektion
}

\section{Ergebnisse}

Bei $49 \%$ der Operationen wurden Drainagen eingelegt. Patienten, bei denen im Rahmen der Operation keine Drainage eingelegt wurde, hatten signifikant weniger Pankreasfisteln als Patienten, bei denen eine Drainage eingelegt wurde $(18 \%$ vs. $27 \%)$, signifikant weniger schwerwiegende Komplikationen (26\% vs. $33 \%$ ), eine kürzere Liegedauer (7 vs. 8 Tage) und weniger Wiederaufnahmen nach Entlassung (20\% vs. $27 \%$ ). Auch die Rate an klinisch relevanten Pankreasfisteln (Notwendigkeit einer interventionellen, endoskopischen oder operativen Maßnahme, chronisch reduzierter Allgemeinzustand, Tod) war bei Patienten ohne Drainage signifikant niedriger (16\% vs. 20\%). Die Raten an Reoperationen, radiologischen Interventionen und die Mortalitätsraten waren bei Patienten mit und ohne prophylaktische Drainageneinlage vergleichbar. Zudem bestanden keine Unterschiede hinsichtlich der wichtigsten Parameter (klinisch relevante Pankreasfisteln, Notwendigkeit radiologischer Interventionen) zwischen den OperateurGruppen „routine drainers“ und „routine non-drainers".

\section{Diskussion}

Die Autoren folgern, dass eine routinemäßige Drainageeinlage nach Pankreasresektionen nicht zu einer Verbesserung der Ergebnisse führt. Insbesondere werde sowohl die Ausbildung von Pankreasfisteln als auch die Notwendigkeit einer postoperativen Intervention durch pro- phylaktische Drainageneinlage nicht verhindert. Anhand der Ergebnisse könnte eine routinemäßige Drainageneinlage die Komplikationsrate sogar erhöhen.

Diese Ergebnisse stellen die gängige Praxis der routinemäßigen Drainageeinlage eindeutig infrage. Die Aussagekraft der Studie ist allerdings limitiert: Die individuelle, nicht standardisierte Entscheidung zur Drainageeinlage durch die Chirurgen könnte zu einem Bias geführt haben mit Selektion risikoreicherer Fälle in die Gruppe mit Drainageneinlage. Zudem ist fraglich, inwieweit die Ergebnisse von 6 Spezialisten aus einem High-volume-Center allgemein anwendbar sind. Zur Beantwortung dieser Fragen ist eine multizentrische, randomisiert kontrollierte Studie notwendig, wie sie derzeit in Deutschland läuft (PANDRA-Studie, ISRCTN 04937707).

\section{Korrespondenzadresse}

PD Dr. O. Strobel

Klinik für Allgemein-, Viszeralund Transplantationschirurgie, Universität Heidelberg, Im Neuenheimer Feld 110, 69120 Heidelberg Oliver.Strobel@med.uni-heidelberg.de

Interessenkonflikt. Keine Angaben den entsprechend der Präferenz der Operateure eingelegt, die in jeweils 2 „routine drainers" (Drainagen in $>95 \%$ ), ,selective drainers" und "routine non-drainers" (Drainagen in $~ 15 \%$ ) eingeteilt wurden. Perioperative Parameter und Morbidität wurden prospektiv erfasst. 